with $2.4 \%$ in the MP group. The 2-year eventfree survival rate was significantly higher in patients who received thalidomide than in those who did not (54\% vs $27 \%$; $P=0.0006)$. A significant overall survival advantage for MPT was observed 9 months after randomization $(P=0.03)$. Thalidomide was, however, associated with a $22.7 \%$ increase in the rate of grade 3-4 toxicities. The incidence of these adverse events was reduced with the introduction of anticoagulant prophylaxis halfway through the trial. In conclusion, MPT could become the regimen of choice in the first-line treatment of older patients with myeloma, but further studies with long-term follow-up are needed to assess survival benefit.

Alexandra King

Original article Palumbo A et al. (2006) Oral melphalan and prednisone chemotherapy plus thalidomide compared with melphalan and prednisone alone in elderly patients with multiple myeloma: randomised controlled trial. Lancet 367 : $825-831$

\section{Multimarker RT-PCR for detection of minimal residual disease in breast cancer patients}

Approximately one-third of patients diagnosed with node-negative breast cancer develop distant metastases within 5 years, but routine histological examination using hematoxilyn and eosin (H\&E) staining has limited sensitivity in detecting these metastases. Using the breast-cancer-specific markers mammaglobin (MGB)-2 and NY-BR-1, and the nonspecific epithelial marker cytokeratin (CK)-19, researchers developed a multimarker reverse transcription polymerase chain reaction (RT-PCR) assay to detect minimal residual disease in lymph nodes of patients with breast cancer.

RNA was extracted from 30 sentinel lymph nodes from 28 patients with histologically confirmed primary breast adenocarcinoma. H\&E staining identified breast cancer metastasis in six nodes $(20.0 \%)$ and immunohistochemistry for CK showed metastasis in eight nodes $(26.7 \%)$, including all those identified by H\&E staining. RT-PCR, however, showed greater sensitivity, with positive results for at least one of the three markers detected in 15 nodes $(50.0 \%)$, including all those identified as positive by the other techniques. RT-PCR was positive for all three markers in seven nodes, for two markers in three nodes and for one marker in five nodes; NY-BR-1 was the most sensitive single marker. Three primary breast cancer specimens used as positive controls showed amplification of each of the three markers; none of the markers were amplified in 13 negative control specimens.

The authors conclude that an RT-PCR assay that probes for MGB-2, NY-BR-1 and CK-19 appears to be more sensitive than standard histological examination or immunohistochemistry. As not all markers are uniformly expressed in all tumors, the multimarker approach is more sensitive than use of single markers, and might in future prove useful in breast cancer staging.

Rebecca Ireland

Original article Nissan A et al. (2006) Multimarker RTPCR assay for the detection of minimal residual disease in sentinel lymph nodes of breast cancer patients. Br J Cancer 94: 681-685 